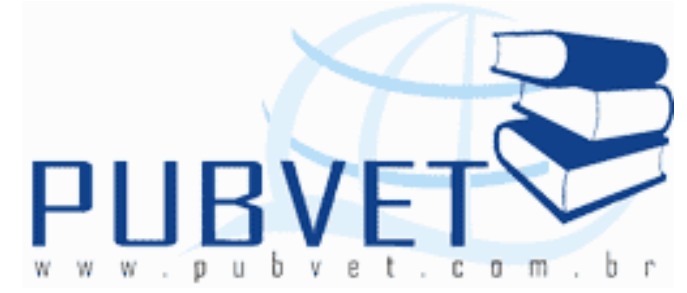

PUBVET, Publicações em Medicina Veterinária e Zootecnia.

\title{
A técnica do ultrassom para avaliação da carcaça em tempo real
}

Keni Eduardo Zanoni Nubiato ${ }^{1}$, Alexandre Rodrigo Mendes Fernandes ${ }^{2}$, Luis Gustavo Castro Alves ${ }^{1}$, José Carlos da Silveira Osório ${ }^{3}$, Maria Teresa Moreira Osório $^{3}$, Camila Magalhães da Cunha ${ }^{1}$, Thatiane da Cunha Cornélio ${ }^{1}$, Ana Paula Catalano Neto ${ }^{1}$

${ }^{1}$ Mestrando(a) do Programa de Pós Graduação em Zootecnia Universidade Federal da Grande Dourados,UFGD, Dourados MS, bolsista Capes;

${ }^{2}$ Docente do Curso de Pós Graduação em Zootecnia - UFGD;

${ }^{3}$ Professor(a) Visitante Nacional Sênior da Universidade Federal da Grande Dourados- UFGD - CAPES; Bolsista Produtividade do CNPq.

\section{Resumo}

Com esta revisão objetivou-se apresentar e consolidar a eficiência da técnica de ultrassom para a avaliação de carcaças em tempo real. Serão abordados os princípios de funcionamento, conceitos da técnica de ultrassom e sua utilização para avaliação de carcaças em tempo real, como técnica de mensuração in vivo. Dentro da produção animal, a técnica vem sendo utilizada para o diagnóstico de certas patologias, detecção de gestação ou de desordens reprodutivas, transferência de embriões e como alternativa inovadora para a mensuração das características de carcaça de animais vivos. Com a crescente preocupação como valor qualitativo da carcaça e a possibilidade de melhoramento genético destas características, esta técnica adquiriu grande 
NUBIATO, K.E.Z. et al. A técnica do ultrassom para avaliação da carcaça em tempo real. PUBVET, Londrina, V. 7, N. 8, Ed. 231, Art. 1526, Abril, 2013.

importância nos estudos de nutrição e composição corporal. Diversas pesquisas comprovam a viabilidade e acurácia da técnica para a avaliação de carcaças em tempo real, principalmente para medição da área de olho de lombo e espessura de gordura de cobertura, muito utilizadas em bovinos e ovinos. Conclui-se então, que a ultrassonografia em tempo real é uma ferramenta economicamente viável e eficaz para ser utilizada dentro dos diversos sistemas de terminação, para a avaliação das carcaças antes do abate dos animais.

Palavras-chave: área de olho de lombo, espessura de gordura de cobertura, medidas in vivo, ruminantes

\title{
The technique of ultrasound for carcass evaluation in real time
}

\begin{abstract}
With this revision aimed to consolidate and present the efficiency of the technique of ultrasound for the evaluation of carcasses in real time. It will examine the principles of operation, concepts of ultrasound technique and its use for evaluation of carcasses in real time, as a technique for measuring in vivo. In animal production, the technique has been used to diagnose certain diseases, detection of pregnancy or reproductive disorders, embryo transfer and as an innovative alternative to the measurement of carcass characteristics in live animals. With growing concern as qualitative value of carcass and the possibility to improve these characteristics, this technique gained great importance in the study of nutrition and body composition. Several studies show the feasibility and accuracy of the technique for the evaluation of carcasses in real time, especially for measurement of rib eye area and fat thickness, much used in cattle and sheep. It follows then that the real time ultrasound is a viable and effective tool to be used within the various finishing systems for the evaluation of carcasses of animals before slaughter.
\end{abstract}

Keywords: fat thickness, in vivo measurements, rib eye area, ruminant 
NUBIATO, K.E.Z. et al. A técnica do ultrassom para avaliação da carcaça em tempo real. PUBVET, Londrina, V. 7, N. 8, Ed. 231, Art. 1526, Abril, 2013.

\section{Introdução}

Fenômeno físico de ocorrência natural, o som, é um dos mais importantes eventos que fazem parte do conjunto da percepção sensitiva do ser humano, e pode ser definido como a propagação de uma vibração em um meio elástico (sólidos, líquidos e gasosos). As propagações de alta frequência ou ultrassom têm servido ao homem no âmbito das ciências médicas ocupando posição importante entre os mais eficazes métodos modernos de diagnósticos não invasivos (Biscegli, 2006). O desenvolvimento desta técnica se tornou importante na produção animal para a avaliação da composição e da qualidade da carcaça em animais vivos e têm mobilizado consideráveis recursos em pesquisas.

Nos últimos anos, o uso da técnica da ultrassonografia na avaliação de carcaças de animais destinados ao abate, principalmente bovinos e ovinos, se intensificou, especialmente por causa da melhoria dos equipamentos. O ultrassom é um equipamento que se tornou valioso para o melhoramento genético animal, pela facilidade de seu manuseio, pelo fornecimento rápido da informação requerida e pela obtenção das medidas diretamente do animal vivo, portanto, sem a necessidade de abate para determinação das características em estudo (Suguisawa, 2002). Sendo assim, com esta revisão objetivou-se apresentar e consolidar a eficiência da técnica de ultrassom para a avaliação de carcaças em tempo real.

\section{Princípios de funcionamento e conceitos da técnica de ultrassom}

A história da tecnologia do ultrassom iniciou-se na segunda guerra mundial, entretanto, este tem sido usado para obter imagens de diagnóstico de tecidos moles, na indústria da produção animal desde meados dos anos 50 . O ultrassom é baseado na emissão de ondas com frequências situadas acima do limite audível pelo ser humano, ou seja, acima de $16 \mathrm{KHz}$. Para a obtenção de imagens são utilizadas frequências entre 1 e 10 MHz (Biscegli, 2006).

A propagação de ondas ocorre em meios líquidos, sólidos e em tecidos biológicos. A velocidade de propagação depende basicamente da interação das 
NUBIATO, K.E.Z. et al. A técnica do ultrassom para avaliação da carcaça em tempo real. PUBVET, Londrina, V. 7, N. 8, Ed. 231, Art. 1526, Abril, 2013.

ondas mecânicas ultrasônicas com o meio. A Tabela 1. apresenta a velocidade de propagação de ondas em função de diferentes meios.

Segundo Augusto e Pachaly (2000), a qualidade da imagem gerada pelo ultrassom e sua correta interpretação dependem do conhecimento das interações entre as ondas e os tecidos ou órgãos que se deseja avaliar.

Tabela 1. Propagação de ondas ultrasônicas em diferentes meios.

\begin{tabular}{lc}
\hline Meio & Velocidade de propagação $(\mathrm{m} / \mathrm{s})$ \\
\hline Ar & 330 \\
Água & 1500 \\
Gordura & 1430 \\
Músculo & 1620 \\
Tecidos moles & 1540 \\
Ossos & 3500
\end{tabular}

Fonte: Adaptado de Biscegli (2006).

O ultrassom sofre reflexão e refração nas interfaces onde ocorre uma mudança na densidade, ao se propagar em um meio e ao passar de um meio para outro. As ondas sofrem atenuação da intensidade do sinal, devido aos efeitos de absorção, reflexão e refração. Reflexão é a propriedade que uma onda sonora tem de refletir quando encontra uma superfície de separação entre dois meios elásticos diferentes. Já a refração é a mudança de velocidade e de direção que sofre a onda sonora ao passar de um meio elástico a outro. A representação desses dois efeitos sofridos pela onda ultrassônica é apresentada na Figura 1. 

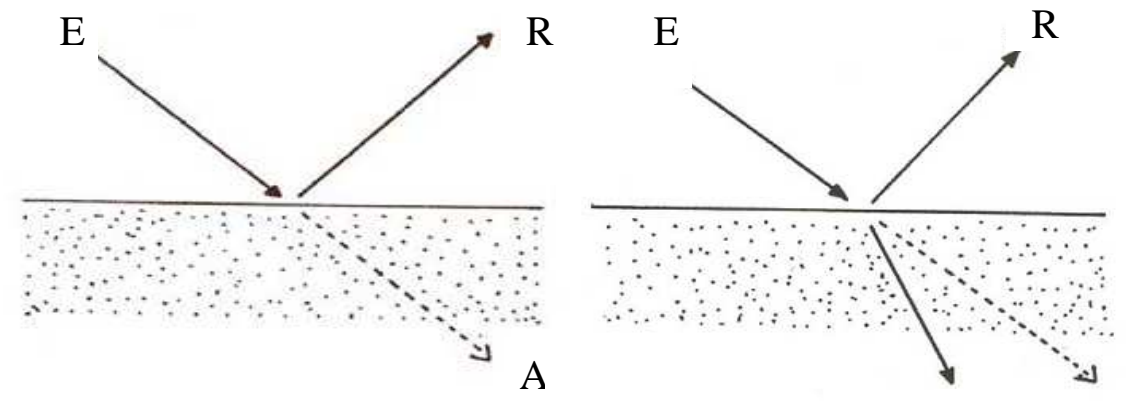

Rf

A meio 1

meio 2

$\mathrm{E}=$ Onda emitida

$\mathrm{R}=$ Onda refletida

$\mathrm{RF}=$ Onda desviada (refração)

$A=$ Onda absorvida

Figura 1. Propagação de ondas ultrassônicas em função de diferentes meios. Adaptado de Biscegli (2006)

A onda ultrassônica é introduzida no meio de propagação através de um elemento emissor que vibra com frequência determinada, a partir do transdutor do aparelho de ultrassom. O elemento emissor é composto por um material piezoelétrico (cristais de quartzo), que apresenta a capacidade de variar suas dimensões físicas e produzir pressões, gerando ondas sonoras de alta frequência. Quando a aplicação de uma força mecânica (onda sonora) na superfície do material resulta no aparecimento de uma voltagem nos eletrodos, temos o efeito piezoelétrico inverso. Esses efeitos permitem a captação das imagens no monitor do aparelho (Biscegli, 2006).

O aparelho de ultrassom basicamente mede a reflexão das ondas de alta frequência que ocorrem quando estas passam através dos tecidos. Após o transdutor ter sido colocado em contato com o animal, o aparelho converte pulsos elétricos em ondas de alta frequência, que ao encontrar diferentes tecidos corpóreos dentro do animal promove uma reflexão parcial (eco) em tecidos menos densos, ou total em tecidos de alta densidade como os ossos. Mesmo após a ocorrência do eco, as ondas de alta frequência continuam a se 
NUBIATO, K.E.Z. et al. A técnica do ultrassom para avaliação da carcaça em tempo real. PUBVET, Londrina, V. 7, N. 8, Ed. 231, Art. 1526, Abril, 2013.

propagar pelo corpo do animal e o conjunto de informações enviadas pelas reflexões transmitidas ao transdutor é projetado em uma tela como imagem, onde as mensurações são realizadas.

A técnica pode ser utilizada para o diagnóstico de certas patologias, deteç̧ão de gestação ou de desordens reprodutivas, transferência de embriões e como alternativa inovadora para a mensuração das características de carcaça de animais vivos (Houghton e Turlington, 1992). Inicialmente utilizava-se o ultrassom de modo $A$, isto é apropriada somente para mensurações lineares como a espessura da camada de gordura subcutânea e profundidade do músculo, envolvendo apenas um transdutor. Posteriormente foi desenvolvido o ultrassom de modo $B$, envolvendo um arranjo linear de vários transdutores. $O$ aparelho de ultrassom em tempo real é a versão do modo $B$ que disponibiliza a imagem instantaneamente. Graças ao desenvolvimento desta metodologia mais acurada, a crescente preocupação como valor qualitativo da carcaça e a possibilidade de melhoramento genético destas características, esta técnica adquiriu grande importância nos estudos de nutrição e composição corporal a partir de 1950 (Hassen et al., 1998).

\section{Avaliação de carcaças}

O ultrassom entra neste contexto como técnica viável, acurada e de custo aceitável para esta função (Houghton e Turlington, 1992). A técnica, utilizada para outros fins, desde a década de 30, pode ser utilizada também para estimar o crescimento de determinados músculos, para predizer a composição da carcaça e o rendimento de cortes comerciais antes do abate, além de poder ser utilizada para estabelecer o escore de condição corporal e definir um estado nutricional (Bruckmaier et al., 1998). Atualmente existe grande interesse no desenvolvimento de sistemas de avaliação e classificação para carcaças bovinas e ovinas. A utilização de instrumentos de alta tecnologia pode proporcionar medições acuradas de componentes da carcaça impossíveis de serem obtidas por avaliações visuais ou palpação do animal vivo. 
Em bovinos e ovinos as principais medidas são a área de olho de lombo $(A O L)$ e a espessura de gordura de cobertura (EGC) na seção do músculo Longissimus lumborum a partir de imagens tomadas entre a $12^{\mathrm{a}}$ e $13^{\mathrm{a}}$ costelas. Estas estimativas, quando obtidas por técnicos experientes, têm apresentado boa repetibilidade, assim como as correlações destas com as medidas correspondentes tomadas na carcaça após o abate dos animais (Hassen et al., 1998).

A Figura 2 apresenta a localização do sítio anatômico de avaliação (secção transversal entre a $12^{\mathrm{a}}$ e $13^{\mathrm{a}}$ costelas e imagem ultrassonográfica).

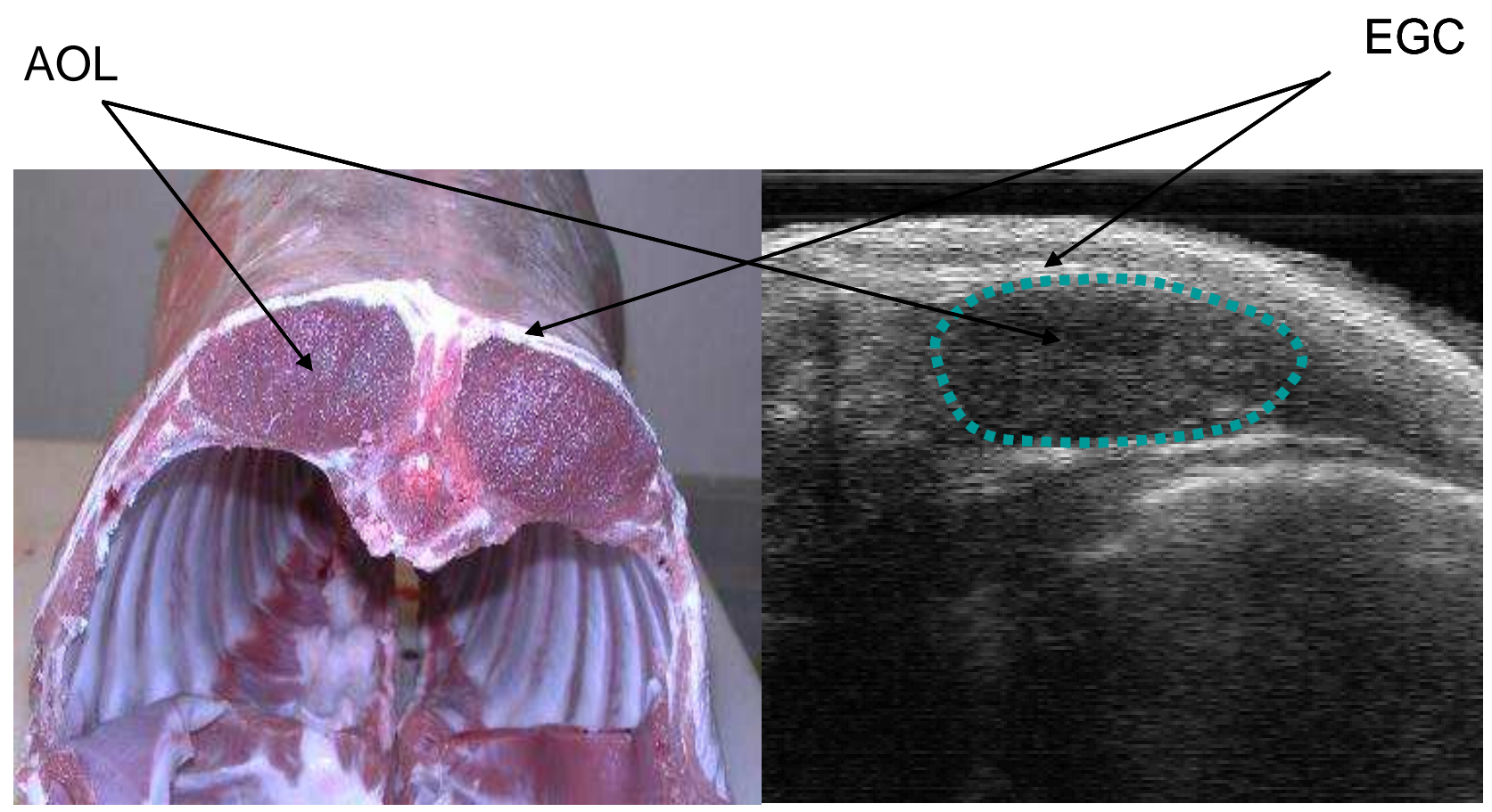

Figura 2. Localização do sítio anatômico de avaliação (secção transversal entre a $12^{\mathrm{a}}$ e $13^{\mathrm{a}}$ costelas e imagem ultrassonográfica tomada in vivo na mesma região, demonstrando a área de olho de lombo ( $A O L)$ e a espessura de gordura de cobertura (EGC)).

Os valores de repetibilidade estimados para as mensurações de AOL e EGC obtidas pela técnica de ultrassom, entre várias tomadas de um mesmo técnico em um animal e também entre medidas de técnicos diferentes no mesmo animal, têm sido altos, demonstrando que em geral as medidas são relativamente fáceis de serem obtidas e que podem ser bastante confiáveis (Bergen et al., 1997). Porém, McLaren et al. (1991) em seus trabalhos 
NUBIATO, K.E.Z. et al. A técnica do ultrassom para avaliação da carcaça em tempo real. PUBVET, Londrina, V. 7, N. 8, Ed. 231, Art. 1526, Abril, 2013.

observaram que os maiores componentes de erros estão ligados aos operadores mal treinados ou quando vários técnicos fazem a leitura e interpretação da imagem.

Dentre os vários fatores apontados como causas das diferenças entre as medidas por ultrassom e aquelas obtidas na carcaça pode-se citar o método de remoção do couro, que retira quantidade variável da camada de gordura da carcaça, o método de suspensão da carcaça que provoca mudanças na sua conformação, o desenvolvimento o rigor mortis, mensuração inadequada da $\mathrm{AOL}$, corte incorreto na seç̧ão da $12^{\mathrm{a}}-13^{\mathrm{a}}$ costelas e o revestimento da camada de gordura da carcaça. Outro fator evidente é que as medições feitas no animal vivo por ultrassom e na carcaça são obtidas em posições muito diferentes, o que compromete as correlações feitas entre as mesmas Brethour, 1992; Bergen et al., 1997).

Em estudos realizados para avaliar a repetibilidade das mensurações Brethour (1992), trabalhou com 580 animais e avaliando-os antes do abate e as carcaças ao final do processo. Este autor concluiu que existiu uma correlação de 0,97 entre as duas medidas. Observou também que animais com gordura de cobertura menor apresentavam maiores diferenças entre as mensurações.

Mercadante et al. (1999) avaliaram por ultrassom, a área de olho de lombo e a espessura de gordura de cobertura de 690 tourinhos com idade entre 9 e 13 meses em prova de ganho de peso, e obtiveram melhores correlações com a idade $\left(r^{2}=0,67\right)$ e peso $\left(r^{2}=0,79\right)$ para as medidas da área de olho de lombo, do que para as medidas da gordura de cobertura. Os autores concluíram que a obtenção da área de olho de lombo por ultrassom, na faixa etária em que os animais se encontravam, pode fornecer informações das mudanças ocorridas neste músculo durante o crescimento dos animais. Ainda na avaliação de machos jovens, Bruckmaier et al. (1998) estudaram a área de olho de lombo e a gordura de cobertura durante o processo de engorda de animais castrados e inteiros e concluíram que a técnica do ultrassom foi eficiente na determinação de diferenças no estado físico e nutricional entre os 
NUBIATO, K.E.Z. et al. A técnica do ultrassom para avaliação da carcaça em tempo real. PUBVET, Londrina, V. 7, N. 8, Ed. 231, Art. 1526, Abril, 2013.

grupos. A Tabela 2 apresenta os coeficientes de correlação encontrados por diversos autores que avaliaram a técnica do ultrassom em relação às medidas realizadas nas carcaças de bovinos e ovinos.

Tabela 2. Correlações entre medidas ultrassonográficas e nas carcaças.

\begin{tabular}{lll}
\hline Autores & AOL & EGC \\
\hline Stouffer et al. (1989) & 0,76 & 0,86 \\
Perry et al.(1990) & 0,90 & 0,96 \\
Bullock et al. (1991) & 0,90 & 0,79 \\
Bergen etal.(1996) & 0,80 & 0,84 \\
May et al.(2000) & 0,61 & 0,81 \\
Silva et al. (2001) & 0,74 & 0,87 \\
\hline
\end{tabular}

A técnica da ultrassonografia pode ser utilizada também para se estabelecer programas alimentares para a terminação do gado de corte, fornecendo informações para se avaliar o número de dias de alimentação e o ponto final de acabamento dos animais (Houghton e Turlington, 1992).

\section{Conclusão}

A ultrassonografia em tempo real é uma ferramenta economicamente viável, eficaz e confiável, podendo ser utilizada dentro dos diversos sistemas de terminação para a avaliação das características das carcaças antes do abate dos animais.

\section{Referências bibliográficas}

AUGUSTO, A.Q.; PACHALY, J.R. Princípios físicos da ultra-sonografia - Revisão bibliográfica. Arquivos de Ciências Veterinárias e Zoologia. v.3, n.1, p.61-65, 2000.

BERGEN, R.D.; McKINNON, J.J.; CHRISTENSEN, D.A. et al. Prediction of lean yield in yearling bulls using real-time ultrasound. Canadian Journal of Animal Science, v.76, p.305-311, 1996.

BERGEN, R.D.; MCKINNON, J.J.; CHRISTENSEN, D.A. et al. Use of the real-time ultrasound to evaluate live animal carcass traits in young performance tested beef bulls. Journal of Animal Science. v. 73, n. 3, p. 2300-2307, 1997.

BISCEGLI, C.I. Conceitos da física do ultrassom. In. Workshop de ultrassonografia para avaliação de carcaças bovinas, Uberaba, 2006, 16p. 
BRETHOUR, J.R. The repetibility and accuracy of ultrasound in measuring backfat of cattle. Journal of Animal Science. v. 70, n. 5, p. 1039-1044, 1992.

BRUCKMAIER, R.M.; LEHMANN, E.; HUGI, W. Ultrasonic measurement of longissimus dorsi muscle and backfat associated with metabolic and endocrine traits, during fattening of intact and castrate male cattle. Livestock Production Science. v. 53, p. 123-134, 1998.

BULLOCK, K.D; BERTRAND, J.K.; BENYSHEK, L.L. et al. Comparison of real-time ultrasound and other live measures to carcass measures as predictors of beef cow energy stores. Journal of Animal Science, v.69, p.3908-3916, 1991.

HASSEN, A.; WILSON,D.E.; WILLHAN,R.L. et al. Evaluation of ultrasound measurement of fat thickness and longissimus muscle area in feedlot cattle: Assessment of accuracy and repeatability. Canadian Journal of Animal Science. v. 78, n. 1, p. 277-285, 1998.

HOUGHTON, P.L.; TURLINGTON, L.M. Application of ultrasound for feeding and finishing animals. Journal of Animal Science. v. 70, n. 4, p. 930-941, 1992.

MAY, S.G.; MIES, W.L.; EDWARDS, J.W. et al. Using live estimates and ultrasound measurements to predict carcass cutability. Journal of Animal Science, v.78, p.1255-1261, 2000.

MCLAREN, D.G.; NOVAKOFSKI, D.F.; PARRETT, L.L. A study of operator effects on ultrasound measures of fat depht and longissimus muscle area in cattle, sheep and pigs. Journal of Animal Science. v. 69, n. 1, p. 54-62, 1991.

MERCADANTE, M.E.Z.; CYRILLO, J.N.S.G.; SILVA, S.L. Medidas de ultrassom da área de olho de lombo e espessura de gordura de cobertura de bovinos em prova de ganho de peso. In: REUNIÃO ANUAL DA SOCIEDADE BRASILEIRA DE ZOOTECNIA, 36., 2001, Porto Alegre. Anais... Porto Alegre, p.138, 1999.

PERRY, T. C., S. J. AINSLIE, M. J. TRAXLER, D. G. FOX, AND J. R.STOUFFER. Use of real-time and attenuation ultrasonic measurements to determine backfat thickness, ribeye area, carcass marbling and yield grade in live cattle. Journal of Animal Science. v.88, n.337. 1990. (Suplemento 1), resumo.

SILVA, S.L.; LEME, P.R.; FIGUEIREDO, L.G.G. et al. Correlações entre características de carcaça obtidas in vivo por ultrassonografia e na carcaça post mortem em novilhos Nelore. In: REUNIÃO DA SOCIEDADE BRASILEIRA DE ZOOTECNIA, 38., Piracicaba, 2001. Anais... Piracicaba: Sociedade Brasileia de Zootecnia, p.1206-1208, 2001.

STOUfFER, J. R., T. C. PERRY, AND D. G. FOX. New Techniques for real time ultrasonic evaluations of beef cattle. Journal of Animal Science. v.67, n.121, 1989. (Suplemento 1), resumo.

SUGUISAWA, L. Ultrassonografia para predição das características e composição da carcaça de bovinos. Dissertação (Mestrado) Piracicaba-SP, 2002. 70p. 